\title{
LXPER Index: A Curriculum-specific Text Readability Assessment Model for EFL Students in Korea
}

\author{
Bruce W. Lee ${ }^{1,2}$ \\ ${ }^{1}$ Research \& Development Center \\ LXPER, Inc. \\ Seoul, South Korea
}

\author{
Jason Hyung-Jong Lee ${ }^{1}$ \\ ${ }^{2}$ Department of Computer \& Information Science \\ University of Pennsylvania \\ Philadelphia, PA, USA
}

\begin{abstract}
Automatic readability assessment is one of the most important applications of Natural Language Processing (NLP) in education. Since automatic readability assessment allows the fast selection of appropriate reading material for readers at all levels of proficiency, it can be particularly useful for the English education of English as Foreign Language (EFL) students around the world. However, most readability assessment models are developed for the native readers of English and have low accuracy for texts in non-native English Language Training (ELT) curriculum. We introduce LXPER Index, which is a readability assessment model for non-native EFL readers in the ELT curriculum of Korea. To measure LXPER Index, we use the mixture of 22 features which we prove to be significant in text readability assessment. We also introduce the Text Corpus of the Korean ELT Curriculum (CoKEC-text), which is the first collection of English texts from a non-native country's ELT curriculum with each text's target grade level labeled. In addition, we assembled the Word Corpus of the Korean ELT Curriculum (CoKEC-word), which is a collection of words from the Korean ELT curriculum with word difficulty labels. Our experiments show that our new model, trained with CoKEC-text, significantly improves the accuracy of automatic readability assessment for texts in the Korean ELT curriculum. The methodology used in this research can be applied to other ELT curricula around the world.
\end{abstract}

Keywords-Natural language processing; machine learning; text readability assessment; EFL (English as Foreign Language) education

\section{INTRODUCTION}

Readability Assessment helps quantify the level of difficulty that a reader experiences in comprehending a certain text. Since automatic readability assessment enables the convenient selection of appropriate reading material for readers with different levels of proficiency [1], readability assessment has been an important field of research since as early as the 1940's [11]. Since then, more than 200 readability formulas were developed [3], but most of them concentrated on the general audience in the United States. We argue that there is a need for the development of an improved text readability assessment method for use in English as Foreign Language

This work is partly supported by the Ministry of SMEs and Startups, Republic of Korea.
(EFL) education around the world.

In China, Japan, and South Korea, many high and middle school students, in addition to their regular classes, also attend English language schools. English education plays an extremely important role in the national educational systems and college entrance exams of the three countries [23], [25]. Furthermore, it is estimated that more than $\$ 3$ billion is spent annually on English education in South Korea, and in Japan, it is much more [23]. Despite the amount of importance put in English education in such countries, the automatic text readability assessment method has not been in active use. This is mostly because of the low level of accuracy of the traditional readability assessment formulas for use in an international EFL curriculum, which we will prove later in this paper.

Previous work in automatic readability assessment has focused on analyzing the generic features of a text. For example, Flesch-Kincaid readability tests use variables like total words, total sentences, and total syllables to identify the difficulty of a text [20]. Such features are essential, but we argue that more curriculum-specific features are required for use in EFL education of non-native students. In addition to the traditional method of calculating generic features of a text like average number of words per sentence, average number of syllables per word, average number of noun phrases per sentence, we model the cognitive characteristics and the expected level of the vocabulary of a user group. Implementing cognitively motivated features, which operate at the discourse level of a text, has proven to be efficient in predicting readability for adults with Intellectual Disabilities (ID) [14], but no research has been conducted using corpora from an EFL curriculum.

Obtaining well-formatted graded corpora is one of the most difficult tasks in conducting modern readability assessment research using Natural Language Processing (NLP) techniques. We managed to collect graded corpora that match the English Language Training (ELT) curriculum in Korea. The results we obtain in this research are mainly based on the Korean ELT curriculum, but the novel techniques and features that we introduce are applicable to any other country with an EFL curriculum.

The contributions of this paper are: (1) we utilize a novel graded corpus of texts from an actual EFL curriculum; (2) we 
present the possibility of using a graded corpus of words (that we manually assembled with the help of 20 English teachers) for curriculum-specific optimization; (3) we test the efficiency of discourse-level text analysis for readability assessment in EFL curriculum; (4) we introduce novel readability assessment features for word-level text evaluation; (5) we evaluate the accuracy of our new model in predicting the readability of texts used in non-native ELT curriculum.

The overall structure of our paper is as followed: Section 2 explains some of the past works related to our topic of study, Section 3 describes our hypothesis and briefly outlines the methods we use in our study, Section 4 describes the two corpora that we built, Section 5 describes our linguistic features used in LXPER Index, Section 6 evaluates our index against other popular readability assessment models, and Section 7 includes some of our future plan to improve the accuracy of LXPER Index.

\section{RELATED WORK}

Many readability metrics are measured by calculating a number of shallow features of a text, which include total words, total sentences, and total syllables [20], [24]. However, as later studies proved, such shallow measures are not directly related to the linguistic components that determine readability [10]. Even though these traditional readability metrics are still being used, they can easily misrepresent the complexity of academic texts [12].

Unlike the readability formulas in the past, most recent studies on text readability assessment using machine learningbased approaches [2], [6], [14]. Reference [29] pioneered the statistical approach to readability assessment but the research stopped at applying simple unigram language models to estimate the grade level of a given text. In contrast, modern readability assessment methods often analyze more than 20 features and explore a broader set of linguistic features [15]. Meanwhile, a variety of machine learning frameworks has been explored, but it was proved that the improvement resulting from changing the framework is smaller than that from optimizing the features [18].

Most work on readability assessment has been directed at estimating the reading difficulty for native English learners. Several efforts in developing automated readability assessment techniques have only emerged since 2007 [32]. Reference [17] proved that grammatical features play a more important role in EFL text readability prediction than native English curriculum. Reference [30] showed that the additional use of lexical features (which we also use in this research) has a significant positive effect on EFL readability assessment. However, the common limitation of the previous research in EFL readability assessment was the use of textual data annotated with the readability levels for native readers of English, not EFL readers. The study of automatic readability assessment for EFL students is still in its early stages.

\section{HYPOTHESIS AND METHODS}

We hypothesize that the accuracy of EFL text readability assessment can be improved by adding entity calculation and curriculum-specific vocabulary features. EFL readers have limited exposure to English compared to native readers. As a result, EFL readers would have to work harder at connecting each entity to a semantic relationship, compared to the average native readers. In addition, we believe that the biggest difference between native text readability assessment and EFL text readability assessment is that EFL students strictly follow the specific national ELT curriculum. Unlike native students who learn English from a variety of primary and secondary sources, most EFL students learn English as a school academic subject. Thus, we believe that the performance of EFL text readability assessment will greatly improve with the consideration of the specific national curriculum.

To test our hypothesis, we used the following methodology. We collected two corpora (explained in detail in Section 4). The first is CoKEC-text (Text Corpus of the Korean ELT Curriculum), which we created by putting together the texts approved or administered by the Korean Ministry of Education (MOE). We collected the texts that appeared in the National Assessment of Educational Achievement (NAEA), College Scholastic Ability Test (CSAT), and government-approved middle school textbooks. The second is CoKEC-word (Word Corpus of the Korean ELT Curriculum). We manually assembled the corpus with the help of 30 teachers with more than 20 years of experience in teaching EFL students in the Korean ELT curriculum. We classified 59529 words that appeared in the Korean ELT curriculum. Both CoKEC-text and CoKECword corpora only contain the texts/words from the official ELT curriculum in Korea. We then analyzed the significance of each feature on CoKEC-text. Finally, we combined the significant features into a linear regression model and experimented with a number of feature combinations.

\section{CORPORA}

To test how our linguistic features measure the readability of a text, we collected two English corpora (CoKEC-text and CoKEC-word). Since our goal is to perform a more accurate readability assessment for EFL texts, an ideal corpus for our research must contain texts from a non-native ELT curriculum - in particular, if such texts are labeled with target grade levels. We are not aware of such texts electronically available, so we have decided to collect the texts ourselves. The texts come from government, online, and commercial sources.

\section{A. Graded Text Corpus: CoKEC-Text}

It is extremely rare to encounter a corpus in which texts are labeled as being at specific levels of readability. The Weekly Reader corpus [31] is one of the only electronically available text corpus with target grade level information, but the corpus is not available anymore since 2012, as the publisher became a part of the Scholastic Corporation. In addition, the corpus was annotated with the readability levels for native readers of English, so such a corpus is not suitable to this research. We had no choice but to build grade annotated corpora ourselves to continue developing LXPER Index.

Our first corpus, which we refer to as CoKEC-text, is a collection of 2760 unique texts that are officially approved or administered by the Korean Ministry of Education. The texts are from NAEA, CSAT, and government-approved textbooks. We have been collecting the texts for about 10 years, from 2010 to 2020. Each text is labeled with its target grade level 
(grade 7: 17 texts, grade 8: 215 texts, grade 9: 80 texts, grade 10: 571 texts, grade 11: 596 texts, grade 12: 590 texts, grade 12.5: 691 texts). Grade 12.5 refers to the English texts that were used in CSAT, which is a college entrance exam for Korean universities. (Korean grades 7 to 12 are for middle and high school students of ages 13 to 19 .)

\section{B. Graded Word Corpus: CoKEC-Word}

The classification of word difficulty has been a field of research for as long as the text readability assessment. Thus, there are a number of electronically available word corpus, which include the British National Corpus, gathered by Lancaster University and Cambridge [4], and the Corpus of Contemporary American English [9]. Even though these corpora do not contain target grade level classification, a number of methods to identify the word difficulty has been explored using the already available corpora [21].

Reference [13] identified the "easier" words by calculating each word's Kucera-Francis frequency in the psycholinguistic dictionary [28]. Like so, most research on word difficulty has been based on the frequency that a certain word is used. This is under the assumption that the more frequently used words are more familiar to the native readers of English. However, such a method is inapplicable to EFL students because most have comparatively limited exposure to English, which is highly dependent on their national ELT curriculum.

To measure the word difficulty intended by a certain ELT curriculum (we chose the Korean ELT curriculum due to the ease of access), we gathered 30 English teachers with more than 20 years of teaching experience in Korea. Our second corpus, CoKEC-word, is a collection of 59529 words that appeared in the Korean ELT curriculum from 2010 to 2020. Out of the 59529 words, 30608 words are classified into six categories (A: suitable for grade 1 to 4 students - 1315 words, B: grade 5 to 8 students -1365 words, C: grade 8 to 9 students - 3103 words, D: grade 9 to 11 students - 5269 words, E: grade 11 to 12 students - 7677 words, F: college students 11879 words). The other 28921 words are unclassified as they are proper nouns or abbreviations. Because we are particularly interested in improving the accuracy of readability assessment for the use of EFL students, we focus on the first section (classified into 6 categories) of CoKEC-word.

\section{LingUistic FEATURES AND READABILITY}

In this section, we describe the set of features that we used for readability assessment. Table I is the list of the features and their code names. The list is divided into simple features, cognitively motivated features, and word difficulty features.

\section{A. Simple Features}

We start by implementing some simple features from Flesch-Kincaid metrics [20]: aWPS (average number of Words per Sentence), aSPW (average number of Syllables per Word), and M3S (percentage of words with more than 3 syllables).

Then we also implemented features from other previous research that have proven to be particularly useful in Machine Learning (ML) text readability evaluation. Reference [26] calculated the following features using the Charniak parser [5]: aNP, aNN, aVP, aAdj, aSBr, aPP, nNP, nNN, nVP, nAdj, aSBr,
nPP (description in Table I). The linguistic features that were identified in [26] are still useful, but there has been a massive improvement in the tree-parsing technology. We used the Berkeley Neural Parser [19], a constituency parser, which proved to identify the linguistic features at a higher accuracy than the Charniak parser.

\section{TABLE I. FEATURES}

\begin{tabular}{|c|l|l|}
\hline Count & Code & Description \\
\hline Simple Features & \multicolumn{2}{|l|}{} \\
\hline 1 & aWPS & average number of Words per Sentence \\
\hline 2 & aSPW & avg num of Syllables per Word \\
\hline 3 & aNP & avg num of Noun Phrases per sentence \\
\hline 4 & aNN & avg num of proper nouns per sentence \\
\hline 5 & aVP & avg num of Verb Phrases per sentence \\
\hline 6 & aAdj & avg num of Adjectives per sentence \\
\hline 7 & aSBr & avg num of Subordinate Clauses per sentence \\
\hline 8 & aPP & avg num of Prepositional Phrases per sentence \\
\hline 9 & M3S & \% of words with more or equal to 3 syllables \\
\hline 10 & nNP & total num of Noun Phrases per sentence \\
\hline 11 & nNN & total num of proper nouns per sentence \\
\hline 12 & nVP & total num of Verb Phrases per sentence \\
\hline 13 & nAdj & total number of Adjectives per sentence \\
\hline 14 & nSBr & total num of Subordinate Clauses per sentence \\
\hline 15 & nPP & total num of Prepositional Phrases per sentence \\
\hline Cognitively-Motivated Features \\
\hline 16 & nUE & total number of Unique Entities \\
\hline 17 & aEM & avg num of Unique Entity mentions per sentence \\
\hline 18 & aUE & avg num of Unique Entities per sentence \\
\hline 19 & nLC & total num of Lexical Chains \\
\hline 20 & aLCw & avg num of Lexical Chains per word \\
\hline 21 & aLCn & avg num of Lexical Chains per noun phrase \\
\hline 22 & aCw & avg num of level C (grade 8-9) words per word \\
\hline 24 & aDw & total num of level C (grade 8-9) words \\
\hline 25 & nDw & total num of level D (grade 9-11) words \\
\hline 26 & aEw & avg num of level E (grade 11-12) words per word \\
\hline 27 & nEw & total num of level E (grade 11-12) words \\
\hline 28 & aFw & avg num of level F (college-level) words per word \\
\hline 29 & nFw & total num of level F (college-level) words \\
\hline & &
\end{tabular}

\section{B. Cognitively-Motivated Features}

The cognitively motivated features used in this research are largely influenced by [14], which proved the usefulness of cognitive features in predicting text readability for adults with Intellectual Disabilities (ID). We believe that EFL students and native adults with ID are similar in the sense that they both face difficulty in the semantic encoding of new information. Among the 10 cognitive features from [14], we implemented 6 features that are applicable to EFL students as well. We test the significance of these features on CoKEC-text in Section 5D.

\section{Word Difficulty Features}

The biggest difference between EFL students and native English readers is that the respective national ELT curriculum is the only exposure to English for most EFL students. EFL students learn new English words step by step in accordance with the curriculum. On the other hand, native English readers learn vocabulary from a variety of sources. Thus, we believe that the curriculum-specific features related to vocabularies can be particularly useful in predicting the text difficulty for EFL students. 
In CoKEC-word, we classified 30608 words into six levels. We focused on the average and total number of vocabularies in levels C, D, E, and F (appropriate for students in grade 8 to college level): $\mathrm{aCw}, \mathrm{nCw}, \mathrm{aDw}, \mathrm{nDw}, \mathrm{aEw}, \mathrm{nEw}, \mathrm{aFw}, \mathrm{nFw}$. In
Section 5D, we prove that some of these features have the high Pearson correlations with the target grade level of texts in the Korean ELT curriculum.

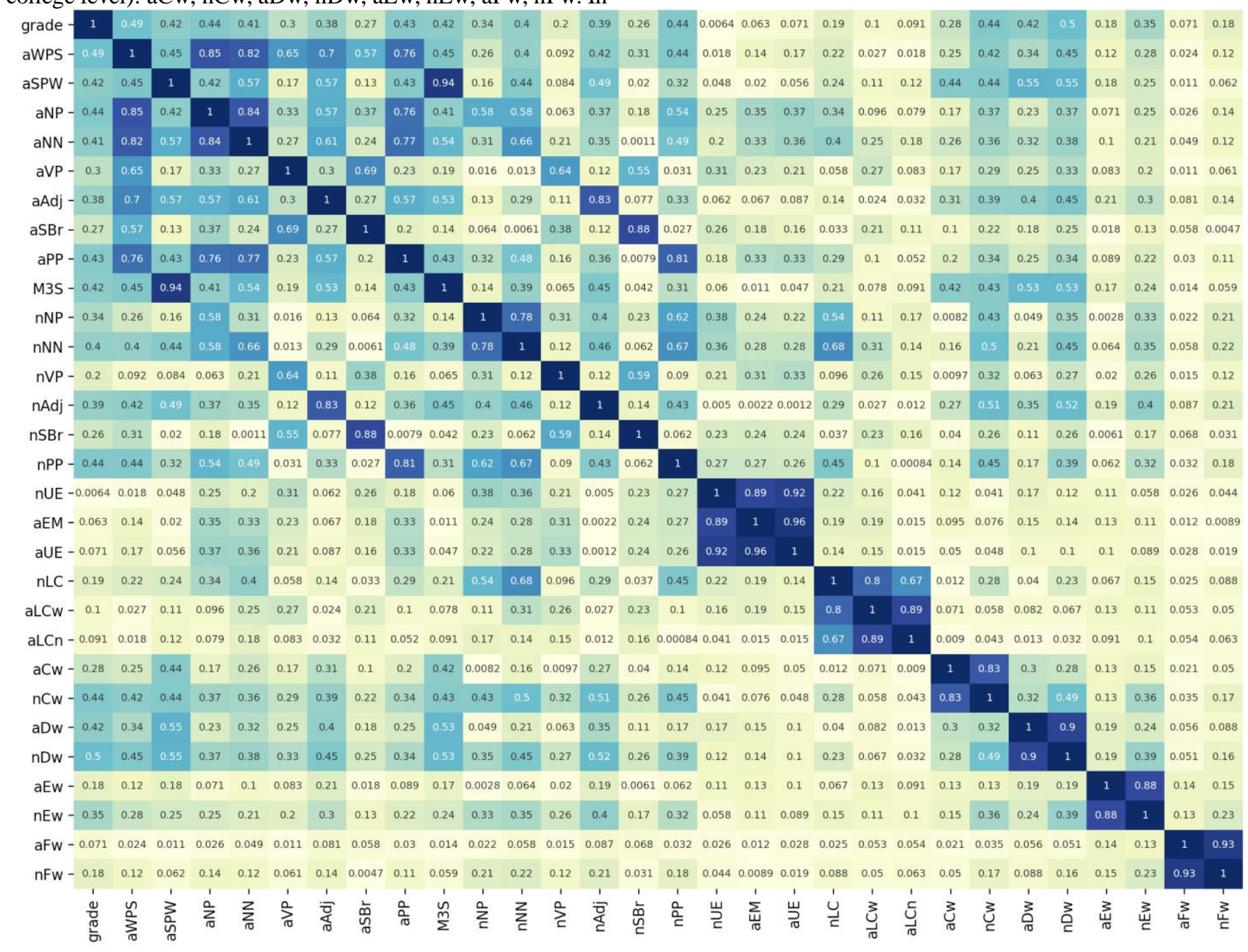

Fig 1. Correlation Heatmap.

\section{Testing the Significance of Features}

To select features to include in our readability assessment model, we analyzed the texts in CoKEC-text to make sure that the results that we get are applicable to a non-native EFL curriculum. We calculated the value of each feature in CoKECtext (the result is shown in Fig. 1), and we used Pearson correlation to test if a feature was significant enough (correlation above 0.05 ) in predicting the target grade level of a text.

The "Cor" column in Table II contains the correlation value of each feature. We put "Yes" in the "Sig" column if the feature was significant enough in predicting the target grade level of a text in CoKEC-text. Meanwhile, "No" means that the feature will not be included in our final readability assessment model because the feature is not significant enough. The only feature that did not show a significant correlation between the feature and the target grade level was the number of Unique Entity (nUE). The lack of significance for nUE can be explained by the repeated mentions of similar entities but less unique entities in higher grade texts.

Moreover, discourse-level features (cognitively motivated features) generally had low correlations compared to simple features and word difficulty features. It can be interpreted that the Korean ELT curriculum puts more emphasis on the difficulty of a sentence structure and the difficulty of each vocabulary, rather than discourse-level analysis.

\section{E. Removing Highly Correlated Features}

To simplify and stabilize our regression model, we decided to remove the features that are highly correlated with each other (correlation above 0.85 ). The highly correlated pairs that we found were: M3S \& aSPW, nSBr \& aSBr, aEM \& nUE, aUE \& nUE, aLCn \& aLCw, nDw \& aDw, nEw \& aEw, nFw $\&$ aFw (also showed in Table II "Pair" column). From each pair, we chose the feature that has a higher correlation with the 
target grade level (in Section 5D): aSPW, aSBr, aEM, aUE, aLCw, nDw, nEw, nFw.

The features that we include in our final readability assessment model are shown in the "Include?" column of Table II. In Table III, the selected features are reorganized in the order of importance (from high to low correlation) in predicting a text's target grade level.

TABLE II. FEATURES SELECTION Process

\begin{tabular}{|c|c|c|c|c|c|}
\hline Count & Code & Cor & Sig & Pair & Include? \\
\hline \multicolumn{6}{|c|}{ Simple Features } \\
\hline 1 & aWPS & 0.494 & Yes & & Yes \\
\hline 2 & aSPW & 0.419 & Yes & M3S & Yes \\
\hline 3 & aNP & 0.445 & Yes & & Yes \\
\hline 4 & aNN & 0.410 & Yes & & Yes \\
\hline 5 & aVP & 0.302 & Yes & & Yes \\
\hline 6 & aAdj & 0.381 & Yes & & Yes \\
\hline 7 & $\mathrm{aSBr}$ & 0.270 & Yes & $\mathrm{nSBr}$ & Yes \\
\hline 8 & aPP & 0.432 & Yes & & Yes \\
\hline 9 & M3S & 0.419 & Yes & aSPW & No \\
\hline 10 & $\mathrm{nNP}$ & 0.342 & Yes & & Yes \\
\hline 11 & $\mathrm{nNN}$ & 0.400 & Yes & & Yes \\
\hline 12 & $\mathrm{nVP}$ & 0.201 & Yes & & Yes \\
\hline 13 & nAdj & 0.392 & Yes & & Yes \\
\hline 14 & $\mathrm{nSBr}$ & 0.261 & Yes & $\mathrm{aSBr}$ & No \\
\hline 15 & $\mathrm{nPP}$ & 0.442 & Yes & & Yes \\
\hline \multicolumn{6}{|c|}{ Cognitively Motivated Features } \\
\hline 16 & nUE & 0.00643 & No & aEM, aUE & No \\
\hline 17 & $\mathrm{aEM}$ & 0.0629 & Yes & nUE & Yes \\
\hline 18 & aUE & 0.0705 & Yes & nUE & Yes \\
\hline 19 & $\mathrm{nLC}$ & 0.190 & Yes & & Yes \\
\hline 20 & $\mathrm{aLCw}$ & 0.10196 & Yes & $\mathrm{aLCn}$ & Yes \\
\hline 21 & aLCn & 0.0912 & Yes & $\mathrm{aLCw}$ & No \\
\hline \multicolumn{6}{|c|}{ Word Difficulty-related Features } \\
\hline 22 & $\mathrm{aCw}$ & 0.280 & Yes & & Yes \\
\hline 23 & $\mathrm{nCw}$ & 0.444 & Yes & & Yes \\
\hline 24 & $\mathrm{aDw}$ & 0.416 & Yes & $\mathrm{nDw}$ & No \\
\hline 25 & $\mathrm{nDw}$ & 0.503 & Yes & $\mathrm{aDw}$ & Yes \\
\hline 26 & $\mathrm{aEw}$ & 0.180 & Yes & $\mathrm{nEw}$ & No \\
\hline 27 & nEw & 0.352 & Yes & $\mathrm{aEw}$ & Yes \\
\hline 28 & $\mathrm{aFw}$ & 0.0714 & Yes & $\mathrm{nFw}$ & No \\
\hline 29 & $\mathrm{nFw}$ & 0.180 & Yes & $\mathrm{aFw}$ & Yes \\
\hline
\end{tabular}

TABLE III. CHOSEN FEATURES IN THE ORDER OF IMPORTANCE

\begin{tabular}{|c|l|l|l|}
\hline Rank & Code & Cor & Type \\
\hline 1 & nDw & 0.503 & Simple Feature \\
\hline 2 & aWPS & 0.494 & Word Difficulty Feature \\
\hline 3 & aNP & 0.445 & Simple Feature \\
\hline 4 & nCw & 0.444 & Word Difficulty Feature \\
\hline 5 & nPP & 0.442 & Simple Feature \\
\hline 6 & aPP & 0.432 & Simple Feature \\
\hline 7 & aSPW & 0.419 & Simple Feature \\
\hline 8 & aNN & 0.410 & Simple Feature \\
\hline 9 & nNN & 0.400 & Simple Feature \\
\hline 10 & nAdj & 0.392 & Simple Feature \\
\hline
\end{tabular}

\begin{tabular}{|l|l|l|l|}
\hline 11 & aAdj & 0.381 & Simple Feature \\
\hline 12 & nEw & 0.352 & Word Difficulty Feature \\
\hline 13 & $\mathrm{nNP}$ & 0.342 & Simple Feature \\
\hline 14 & $\mathrm{aVP}$ & 0.302 & Simple Feature \\
\hline 15 & $\mathrm{aCw}$ & 0.280 & Word Difficulty Feature \\
\hline 16 & $\mathrm{aSBr}$ & 0.270 & Simple Feature \\
\hline 17 & $\mathrm{nVP}$ & 0.201 & Simple Feature \\
\hline 18 & $\mathrm{nLC}$ & 0.190 & Cognitively-Motivated Feature \\
\hline 19 & $\mathrm{nFw}$ & 0.180 & Word Difficulty Feature \\
\hline 20 & $\mathrm{aLCw}$ & 0.101 & Cognitively-Motivated Feature \\
\hline 21 & $\mathrm{aUE}$ & 0.0705 & Cognitively-Motivated Feature \\
\hline 22 & aEM & 0.0629 & Cognitively-Motivated Feature \\
\hline
\end{tabular}

\section{READABILITY ASSESSMENT}

After testing the significance of linguistic features and removing the highly correlated features, we used a linear regression model and trained it with CoKEC-text to build a readability assessment tool; our model is implemented using Python [27]. To evaluate the model's usefulness for EFL students in the ELT curriculum of Korea, we prepared a separate test corpus. The first part of our test corpus is from the official mock tests (pronounced "moi-go-sa" in Korean), used by the Korea Institute of Curriculum \& Evaluation to assess educational achievement of high school students in 2019. There are 264 texts in the first part of our test corpus (grade 10: 88 texts, grade 11: 88 texts, grade 12: 88 texts). The second part of our corpus is from the government-approved middle school textbooks (grade 9: 79 texts). We intentionally collected the texts from two different sources to test how our readability assessment model works on different types of texts. Table IV shows the average number of words per text (aWPT), average number of sentences per text (aSPT), and average number of words per sentence (aWPS) for each grade level in the test corpus.

Next, we calculated the average error for each version of LXPER Index to choose which combination to use. Then we compared LXPER Index with five other popular traditional assessment models: Flesch-Kincaid grade level [20], ColemanLiau Index [8], Dale-Chall Readability Score [11], Coh-Metrix EFL Index [16], and Lexile Measure [22].

\section{A. Versions}

We implemented seven versions of our readability assessment model, which are organized in Table V. The first uses only the simple features, which were studied extensively in previous research (aWPS, aSPW, aNP, aNN, aVP, aAdj, aSbr, aPP, nNP, nNN, nVP, nAdj, nPP). Meanwhile, the second version implements only the cognitively motivated features, which were proved to be useful for readability assessment on adults with ID but haven't been tested on EFL students [14] (aEM, aUE, nLC, aLCw). The third version implements the word difficulty features, which are our novel features in the readability assessment for EFL students $(\mathrm{aCw}$, $\mathrm{nCw}, \mathrm{nDw}, \mathrm{nEw}, \mathrm{nFw}$ ). The fourth version uses both simple features and cognitively motivated features. The fifth uses cognitively motivated features and word difficulty features, while the sixth version uses simple features and word difficulty features. The seventh version combines all the sets of features. These versions are organized in the "Version" column of Table $\mathrm{V}$ as well. 
TABLE IV. TEST CORPUS

\begin{tabular}{|l|l|l|l|l|l|}
\hline Description & Gr 9 & Gr 10 & Gr 11 & Gr 12 & All \\
\hline aWPT & 111.725 & 158.114 & 164.613 & 170.126 & 151.145 \\
\hline aSPT & 14.275 & 8.682 & 9.409 & 9.229 & 10.398 \\
\hline aWPS & 7.826 & 16.599 & 17.495 & 18.432 & 15.088 \\
\hline
\end{tabular}

TABLE V. TESTING COMBINATIONS

\begin{tabular}{|l|l|l|l|l|l|}
\hline Version & Gr 9 & Gr 10 & Gr 11 & Gr 12 & AvgEr* \\
\hline S & 9.817 & 11.055 & 11.385 & 11.639 & 0.655 \\
\hline CM & 10.478 & 11.019 & 11.361 & 11.397 & 0.865 \\
\hline WD & 10.039 & 10.913 & 11.402 & 11.607 & 0.685 \\
\hline S\&CM & 9.727 & 11.056 & 11.395 & 11.634 & 0.636 \\
\hline CM\&WD & 9.894 & 10.927 & 11.455 & 11.616 & 0.665 \\
\hline S\&WD & 9.706 & 10.995 & 11.404 & 11.701 & 0.601 \\
\hline S\&CM\&WD & 9.629 & 10.995 & 11.423 & 11.693 & 0.589 \\
\hline
\end{tabular}

By building seven versions of our model, we can check if there's any certain combination that reduces the assessment error as much as possible. We can also measure the relative impact of implementing our word difficulty features.

Table $\mathrm{V}$ summarizes the average prediction results of our model for texts with different target grade levels. The average error value of our model decreased more than 0.05 grade level by adding the Word Difficulty features, compared to using only the simple features.

\section{LXPER Index}

paragraph1: 11.314610668825809 paragraph2: 11.325568918902054 paragraph3: 11.456926496551013 paragraph4: 11.831492706888628 paragraph5: 12.555917022434922 paragraph6: 11.860643021177644 paragraph7: 12.640597884093928 paragraph8: 11.655660604408641 paragraph9: 13.041646893081829 paragraph10: 12.31356683819909 paragraph11: 12.23811585459722 paragraph12: 10.741342273960948 paragraph13: 12.636533803957006 paragraph14: 12.012046343461847 paragraph15: 11.679116422326873 paragraph16: 10.075361362432751 paragraph17: 10.218809526336205 paragraph18: 11.738985102882594 paragraph19: 10.65425538041245 paragraph20: 11.626751141441773

average: 11.680897413318661 standard dev.: 0.804484551560757

Fig 2. Sample Output.
As we can see from Table III and Table V, cognitively motivated features do not seem to have as much effect as we expected in Section 3. Our explanation is that the non-native ELT curriculum simply puts more focus on the difficulty of words than discourse-level analysis. Even though the cognitively motivated features' correlation with target grade level is not as strong as we hypothesized, it seems that cognitively motivated features do improve the accuracy of our model after a few tests - including the one in Table V. We decided to move on with all three categories of features. The sample output of LXPER Index is shown in Fig. 2.

\section{B. Comparison with Other Readability Tools}

Like we did in Section 6A, we trained our regression models on CoKEC-text and tested it on the separate test corpus that we prepared. By doing so, we could make sure that our model is working properly on the texts from outside of CoKEC-text.

To comparatively evaluate how our model performs in measuring the target grade level of a text in the Korean ELT curriculum, we ran the same test on five other popular metrics. Using Python [27], we created calculator programs for FleschKincaid [20], Coleman-Liau [8], and Dale-Chall [11] formulas. We used the electronically available tools (from the official source) to calculate Lexile Measure [22] and Coh-Metrix EFL Readability Index [7].

Lexile Measure and Coh-Metrix Index are built based on their unique scales. We initially wanted to rescale Lexile Measure and Coh-Metrix Index and compare all the models in one table, but such comparison could potentially misrepresent the intended results by the initial authors. Thus, we decided to create a separate table for Lexile and Coh-Metrix Index without rescaling. The results are organized in Table VI and Table VII. Columns Gr 9, Gr 10, Gr 11, and Gr 12 contain the average readability predictions of each assessment model for the specific part of our test corpus.

Flesch-Kincaid, Coleman-Liau, and Dale-Chall assessment models are almost entirely dependent on the shallow features like average number of words per sentence and average number of syllables per word. Meanwhile, the grade 10, grade 11 , and grade 12 texts in our test corpus had similar aWPT, aSPT, and aWPS, as shown in Table III. As a result, the three assessment models fail to clearly distinguish the readability levels in grade 10 to grade 12 range in Table VI. On the other hand, the values show a sudden drop when predicting grade 9 part of our test corpus, which is also the part where aWPT, aSPT, and aWPS change drastically.

TABLE VI. TeSTING AgAinst OTHER Models

\begin{tabular}{|l|l|l|l|l|l|}
\hline Model & Gr 9 & Gr 10 & Gr 11 & Gr 12 & AvgEr* \\
\hline Flesch-Kincaid & 5.625 & 10.572 & 9.636 & 9.207 & 2.026 \\
\hline Coleman-Liau & 6.679 & 9.876 & 10.183 & 10.101 & 1.290 \\
\hline Dale-Chall & 5.476 & 7.924 & 7.562 & 7.312 & 3.432 \\
\hline LXPER & 9.629 & 10.995 & 11.423 & 11.693 & 0.589 \\
\hline
\end{tabular}


TABLE VII. TESTING AgAinst OTHER MODELS (LEXILE, COH-METRIX)

\begin{tabular}{|l|l|l|l|l|}
\hline Model & Gr 9 & Gr 10 & Gr 11 & Gr 12 \\
\hline Lexile $\left(\sim \mathrm{Gr}^{*}\right)$ & $644(\sim \mathrm{Gr} 3)$ & $1064(\sim \mathrm{Gr} 6)$ & $\begin{array}{l}1260(\sim \mathrm{Gr} \\
10)\end{array}$ & $\begin{array}{l}1120(\sim \mathrm{Gr} \\
7)\end{array}$ \\
\hline Coh-Metrix & 15.432 & 23.725 & 13.134 & 12.462 \\
\hline LXPER & 9.629 & 10.995 & 11.423 & 11.693 \\
\hline
\end{tabular}

*Expected Grade Level (according to Lexile website, $50^{\text {th }}$ percentile, EOY Spring)

Dale-Chall Readability Score has a variable relating to the number of difficult words. Reference [11] collected a list of 768 words, labeled as "Difficult" or "Not Difficult" by surveying native fourth-grade students. It seems that the formula is unable to identify the difficult words for EFL students, which led to the overall prediction being lower than the target grade level.

Lexile Measure fails to show a constantly increasing trend from grade 9 to grade 12 parts of our test corpus. We believe that this is mostly because it is optimized for native students in the United States. The points where the specific national ELT curriculum defines the "difficulty" of a text are varied. The expected grade level in Table VII is estimated according to a graph on the Lexile website [22]. We followed their standards for the $50^{\text {th }}$ percentile, End of Year (EOY) Spring values.

Coh-Metrix EFL Readability Index, out of the five models that we compared to LXPER Index, is the only model specifically designed for EFL students. It works under the assumption that psycholinguistic and cognitive features have great predictive power in readability assessment for EFL students [10]. The Coh-Metrix EFL Index is almost completely consisted of cognitive features. However, we have shown in Table III that they are not very important in readability assessment for EFL students - at least in the case of the Korean ELT curriculum. In Table VI, Coh-Metrix EFL Index shows a sudden decrease (higher values indicate easier-to-read passages) in the grade 9 section of our test corpus. We believe that this is mostly because the Korean middle school ELT curriculum (grade 7 to 9) mostly consists of conversationbased passages, where a lot of names and locations appear. This led to a sudden increase in the number of entities in the part of the corpus.

LXPER Index was the only assessment model that showed a continuously increasing pattern from grade 9 to grade 12 . LXPER Index, with simple features, cognitively motivated features, and word difficulty features all combined, predicts the target grade level of texts in the Korean ELT Curriculum to within 0.589 grade levels on average.

\section{CONCLUSION}

There have been several attempts to perform automatic readability assessment for EFL students, but the common limitation was that most past research was based on corpora with target grade levels for native students. In this research, we created LXPER Index, a readability assessment model that incorporates simple, cognitive, and word difficulty features and trained the model on CoKEC-text. As a result, we obtained a much more accurate EFL text readability prediction compared to the other assessment models available now.
We also proved the importance of word difficulty features in an EFL curriculum. On the other hand, cognitive features were not as highly correlated to a text's target grade level as we expected. Most importantly, LXPER Index was the only assessment model that showed a continuously increasing pattern from grade 9 to grade 12 in Table VI, which, we believe, is a significant achievement. The average error of 0.589 grade levels was a better performance than other assessment models, but we believe that the accuracy can be improved with further research.

In our future research, we believe that we can improve the accuracy of our model by implementing grammatical features. The size of CoKEC-text is another part that we should work on. CoKEC-text is currently the biggest collection of texts of the Korean ELT curriculum, but LXPER Index's accuracy can be improved with even more texts. The grade coverage is currently from grade 7 to grade 12 . Including texts for lower grades is an approach that we should give an attempt.

We can also attempt regression techniques like logistic regression. In this research, we were not completely sure whether to consider the grade level label in CoKEC-text as a continuous variable or a categorical variable. It is possible that the differences in readability among the texts intended for grade $9,10,11,12$ are uneven. Even though we achieved a comparatively good average error value with linear regression, it might be improved by considering the grade level as a categorical variable.

\section{ACKNOWLEDGMENT}

We wish to thank Dr. Inhwan Lee, Eunsoo Shin, Sangjo Park, Donghyun Lee, Daekyung Kim, Cheongho Jeong, Chanwoo Kim, Dongjun Lee, Hun Heo, Kiman Kim, Jieyeon Seo, and Jihye Jeong for their inputs in this project. This research is partly funded by the Fourth Industrial Revolution R\&D Program, Ministry of SMEs and Startups, Republic of Korea.

\section{REFERENCES}

[1] Alhawiti, K.M. 2014. Natural Language Processing and its Use in Education. International Journal of Advanced Computer Science and Applications, 5(12), 72-76

[2] Aluisio, S., Specia, L., Gasperin, C., Scarton, C. 2010. Readability Assessment for Text Simplification. Proceedings of the NAACL HLT 2010 Fifth Workshop on Innovative Use of NLP for Building Educational Applications, 1-9

[3] Benjamin, R.G. 2012. Reconstructing readability: Recent developments and recommendations in the analysis of text difficulty. Educational Psychology Review, 24(1), 63-88

[4] British National Corpus. 2014. http://corpora.lancs.ac.uk/bnc2014/

[5] Charniak, E. 2000. A maximum-entropy-inspired parser. Proceedings of the 1st Meeting of the NAACL.

[6] Chen, X. and Meurers, D. 2017. Word frequency and readability: Predicting the text-level readability with a lexical-level attribute. Journal of Research in Reading, 41(3), 486-510

[7] Coh-Metrix 3.0. 2017. http://141.225.61.35/cohmetrix2017

[8] Coleman, M. and Liau, T.L. 1975. "A Computer Readability Formula Designed for Machine Scoring," Journal of Applied Psychology, 60(2) 283-284

[9] Corpus of Contemporary American English. 2020. https://www.englishcorpora.org/coca/ 
[10] Crossley, S.A., Greenfield, J. \& McNamara, D.S. 2008. Assessing text readability using cognitively based indices. TESOL Quarterly, 42(3), 475-493

[11] Dale, E. and Chall, J.S. 1949. The concept of readability. Elementary English, 26(23)

[12] Davison, A. and Kantor, R. 1982. On the failure of readability formulas to define readable texts: A case study from adaptations. Reading Research Quarterly, 17(2), 197-209

[13] Devlin, S. and Tait, J. 1998. The use of a psycholinguistic database in the simplification of text for aphasic readers. Linguistic Databases, 161173

[14] Feng, L., Elhadad, N., and Huenerfauth, M. 2009. Cognitively Motivated Features for Readability Assessment. Proceedings of the $12^{\text {th }}$ Conference of the European Chapter of the ACL, 229-237

[15] Feng, L., Jansche, M., Huenerfauth, M., Elhadad, N. 2010. A Comparison of Features for Automatic Readability Assessment. Proceedings of the $23^{\text {rd }}$ International Conference on Computational Linguistics, 276-284

[16] Graesser, A.C., McNamara, D.S., Lourwerse, M.M., and Cai, Z. 2004. Coh-Metrix: Analysis of text on cohesion and language. Behavior Research Methods, Instruments, \& Computers, 36, 193-202

[17] Heilman, M., Collins-Thompson, K., Callan, J., Eskenazi, M. 2007. Combining lexical and grammatical features to improve readability measures for first and second language text. Proceedings of North American Chapter of the Association for Computational Linguistics: Human Language Technologies, 460-467

[18] Kate, R.J., Luo, X., Patwardhan, S., Franz, M., Florian, R., Mooney, R.J., Roukos, S. and Welty, C. 2010. Learning to predict readability using diverse linguistic features. Proceedings of the $23^{\text {rd }}$ International Conference on Computational Linguistics, 546-554

[19] Kitaev, N., and Klein, D. 2018. Constituency Parsing with a selfattentive encoder. Proceedings of the 56th Annual Meeting of the Association for Computational Linguistics, 1, 2676-2686
[20] Kincaid, J.P., Fishburne, R.P., Rogers, R.L., and Chissom, B.S. 1975. Derivation of new readability formulas (automated readability index, fog count, and flesch reading ease formula) for Navy enlisted personnel. Research Branch Report, 8-75

[21] Kucera, H. and Francis, W. 1967. Computational analysis of present-day American English. University Press of New England

[22] Lexile. 2020. https://lexile.com/

[23] Mckay, S.L. 2002. Teaching English as an International Language: Rethinking Goals and Perspectives. OUP Oxford

[24] McLaughlin, G.H. 1969. SMOG grading - a new readability formula. Journal of Reading, 12(8), 639-636

[25] Park, J.K. 2014. Teaching English as a Global Language in Korea: Curriculum Rhetoric and Reality. Asian Englishes, 12(1), 124-129

[26] Peterson, S.E., and Ostendorf, M. 2009. A machine learning approach to reading level assessment. Computer Speech and Language, 23: 89-106

[27] Python Software Foundation. Python Language Reference, version 3.7. Available at http://www.python.org

[28] Quinlan, P. 1992. The Oxford psycholinguistic database, Oxford University Press

[29] Si, L. and Callan, J. 2001. A statistical model for scientific readability. Proceedings of the tenth international conference on Information and knowledge management, 574-576

[30] Vajjala, S. and Meurers, D. 2014. Readability assessment for text simplification: From analyzing documents to identifying sentential simplification. International Journal of Applied Linguistics, 165(2), 194222

[31] Weekly Reader. 2008. http://www.weeklyreader.com

[32] Xia, M., Kochmar, E., Briscoe, T. 2016. Text Readability Assessment for Second Language Learners. Proceedings of the $11^{\text {th }}$ Workshop on Innovative Use of NLP for Building Educational Applications, 12-22 